\title{
Impaired myocardial perfusion is associated with extracellular volume expansion, disease activity and impaired strain and strain rate in systemic sclerosis: a cardiovascular magnetic resonance study
}

\author{
Ntobeko A Ntusi ${ }^{1,2^{*}}$, Emily Sever ${ }^{1}$, Joseph Lockey ${ }^{1}$, Jane M Francis ${ }^{1}$, Stefan K Piechnik', Vanessa M Ferreira ${ }^{1}$, \\ Paul M Matthews ${ }^{3}$, Paul B Wordsworth ${ }^{4}$, Stefan Neubauer ${ }^{1}$, Theodoros D Karamitsos ${ }^{1}$
}

From 18th Annual SCMR Scientific Sessions

Nice, France. 4-7 February 2015

\section{Background}

Systemic sclerosis ( $\mathrm{SSc}$ ) is characterised by vascular dysfunction and multi-organ fibrosis, with the heart commonly involved. Cardiovascular disease (CVD) in SSc may be direct or indirect, but often remains subclinical. SSc patients with apparent cardiovascular clinical features are at greater risk of deterioration and premature cardiovascular death, often from complications of myocardial ischaemia. CMR first-pass perfusion detects myocardial ischaemia with great accuracy. We hypothesised that CMR first-pass perfusion would be able to differentiate between segmental (indicating epicardial coronary artery disease) and non-segmental subendocardial (indicating microvascular dysfunction) perfusion defects in patients with SSc; and that microvascular dysfunction (relating to chronic myocardial inflammation) was more frequent in SSc.

\section{Methods}

17 SSc patients (16 female, mean age $55 \pm 9$ years) and 17 matched controls ( 16 female, mean age $54 \pm 10$ years) were enrolled. All patients with known cardiovascular disease were excluded. Study participants underwent CMR at $1.5 \mathrm{~T}$ and the assessments included cine, tagging, T1 mapping, T2-weighted, perfusion, late gadolinium imaging $\left(0.15 \mathrm{mmol} / \mathrm{kg}\right.$ gadoderic acid - Dotarem $\left.{ }^{\circledR}\right)$ and ECV quantification. Comorbid status, disease activity index (VDAI

'OCMR, Division of Cardiovascular Medicine, University of Oxford, Oxford, UK Full list of author information is available at the end of the article score) and duration of disease were recorded for each subject.

\section{Results}

Myocardial perfusion reserve index was $1.5 \pm 0.3$ and $2.0 \pm 0.4(\mathrm{p}<0.001)$ in SSc and controls, respectively. Non-segmental (circumferential) subendocardial perfusion defects were seen in $41 \%$ of SSc and none $(\mathrm{p}<0.001)$ of controls studied. There was no significant difference in LV size, mass and ejection fraction between SSc patients and controls. Peak systolic circumferential strain and peak diastolic strain rate were impaired in patients. Impaired MPRI correlated with peak systolic strain ( $\mathrm{R}$ $0.91, \mathrm{p}<0.001)$ and peak diastolic strain rate $(\mathrm{R} 0.56$, $\mathrm{p}<0.001)$ in SSc. Furthermore, abnormal MPRI correlated with VDAI ( $\mathrm{R}-0.58, \mathrm{p}=0.02)$ and $\mathrm{ECV}(\mathrm{R}-0.56, \mathrm{p}=0.04)$ in SSc.

\section{Conclusions}

Myocardial perfusion is impaired in asymptomatic SSc patients with apparently normal hearts. Abnormal perfusion correlates with strain, strain rate, disease activity and ECV in SSc. CMR can detect subclinical cardiovascular involvement in SSc.

\section{Funding}

This study was funded by investigator-led grants from Guerbet and GlaxoSmithKline. 
Table 1 Baseline characteristics of SSc patients and controls

\begin{tabular}{|c|c|c|c|}
\hline & Controls $\mathrm{N}=17$ & $\mathrm{SSC} N=17$ & $P$ value \\
\hline Female sex, n (\%) & $16(94)$ & $16(94)$ & 1.00 \\
\hline Age, years & $55 \pm 9$ & $54 \pm 10$ & 0.76 \\
\hline Hypertension, n (\%) & $1(6)$ & $4(24)$ & 0.34 \\
\hline Diabetes, n (\%) & 0 & 0 & - \\
\hline Hyperlipidaemia, n (\%) & $2(12)$ & $3(18)$ & 0.63 \\
\hline $\mathrm{BMl}, \mathrm{kg} / \mathrm{m} 2$ & $24 \pm 4$ & $27 \pm 7$ & 0.21 \\
\hline Methotrexate, n (\%) & $\mathrm{N} / \mathrm{A}$ & $5(29)$ & - \\
\hline Chloroquine, n (\%) & N/A & $1(6)$ & - \\
\hline Leflunomide, n (\%) & N/A & $1(6)$ & - \\
\hline Prednisolone, n (\%) & N/A & $1(6)$ & - \\
\hline NSAID, n (\%) & N/A & $3(18)$ & - \\
\hline HRT/OCP & $3(18)$ & $3(18)$ & 1.00 \\
\hline mRSS & $\mathrm{N} / \mathrm{A}$ & $16 \pm 6$ & - \\
\hline VDAI & N/A & $4(2-5)$ & - \\
\hline ESR, mm/hr (median, IQR) & N/A & $11(8-15)$ & - \\
\hline CRP, mg/L (median, IQR) & $1(1-2)$ & $6(3-11)$ & $<0.001$ \\
\hline Duration of SSc, years (median, IQR) & N/A & $13(7-16)$ & - \\
\hline Duration of DMARDs, years (median, IQR) & $\mathrm{N} / \mathrm{A}$ & $4(2-5)$ & - \\
\hline
\end{tabular}

Continuous data are mean \pm SD unless otherwise indicated.

Categorical data are frequency (percent) unless otherwise indicated.

BMI, body mass index; CRP, C-reactive protein; DMARD, disease modifying anti-rheumatic drug(s); ESR, erythrocyte sedimentation rate; HRT/OCP, hormone replacement therapy or oral contraceptive pill; IQR, interquartile range; mRSS, modified Rodnan skin score; NSAID, non-steroidal anti-inflammatory drug(s); SSc, systemic sclerosis; VDAl, Valentini disease activity index

Table 2 Myocardial structure, function and perfusion in SSc patients and controls

\begin{tabular}{|c|c|c|c|}
\hline & Controls $\mathrm{N}=17$ & $\mathrm{SSC} N=17$ & $P$ value \\
\hline LVEDV indexed to BSA, $\mathrm{ml} / \mathrm{m} 2$ & $78 \pm 17$ & $74 \pm 11$ & 0.15 \\
\hline LVESV indexed to BSA, $\mathrm{ml} / \mathrm{m} 2$ & $21 \pm 5$ & $18 \pm 5$ & 0.06 \\
\hline LVEF, \% & $74 \pm 6$ & $73 \pm 5$ & 0.36 \\
\hline LV Mass indexed to BSA, $\mathrm{g} / \mathrm{m} 2$ & $51 \pm 12$ & $51 \pm 8$ & 0.89 \\
\hline LA size, $\mathrm{mm}$ & $28 \pm 5$ & $37 \pm 6$ & $<0.001$ \\
\hline Mid SA circumferential strain & $-18.7 \pm 1.0$ & $-17.0 \pm 1.7$ & $<0.001$ \\
\hline Peak diastolic circumferential strain rate $(\mathrm{s}-1)$ & $114 \pm 16$ & $86 \pm 24$ & $<0.001$ \\
\hline Presence of LGE (\%) & 0 & $10(59)$ & - \\
\hline Volume fraction of LGE>2SD (\%) & 0 & $2.6 \pm 0.3$ & - \\
\hline Global myocardial T2 SI Ratio & $1.5 \pm 0.2$ & $1.7 \pm 0.4$ & 0.08 \\
\hline Volume fraction of oedema by T2 (\%) & 0 & $18(7-23)$ & - \\
\hline Average myocardial T1, ms & $958 \pm 22$ & $1,008 \pm 28$ & $<0.001$ \\
\hline Volume fraction of T1>990ms (\%) & 0 & $59(43-71)$ & - \\
\hline ECV (\%) & $27.9 \pm 2.4$ & $35.4 \pm 4.7$ & $<0.001$ \\
\hline Rest RPP & $7,989 \pm 1,280$ & $8,478 \pm 1,829$ & 0.37 \\
\hline Stress RPP & $11,732 \pm 1,789$ & $11,980 \pm 1,790$ & 0.69 \\
\hline$\overline{M P R I}$ & $2.0 \pm 0.4$ & $1.5 \pm 0.3$ & $<0.001$ \\
\hline Proportion of non-segmental perfusion defects (\%) & 0 & $7(41)$ & - \\
\hline
\end{tabular}

Continuous data are mean \pm SD unless otherwise indicated.

ECV, extracellular volume; LA, left atrium; LGE, late gadolinium enhancement; LV, left ventricle/ventricular; LVEDV, left ventricular end-diastolic volume; LVEF, left ventricular ejection fraction; LVESV, left ventricular end-systolic volume; MPRI, myocardial perfusion reserve index; RPP, rate pressure product; SA, short axis; SI, signal intensity, SSc, systemic sclerosis 


\section{Authors' details}

'OCMR, Division of Cardiovascular Medicine, University of Oxford, Oxford, UK

${ }^{2}$ Division of Cardiology, Department of Medicine, University of Cape Town,

Cape Town, South Africa. ${ }^{3}$ Division of Brain Sciences, Imperial College

London, London, UK. ${ }^{4}$ Bortnar Institute, Nuffield Department of

Orthopaedics, Rheumatology and Musculoskeletal Sciences, University of

Oxford, Oxford, UK.

Published: 3 February 2015

doi:10.1186/1532-429X-17-S1-Q71

Cite this article as: Ntusi et al:: Impaired myocardial perfusion is associated with extracellular volume expansion, disease activity and impaired strain and strain rate in systemic sclerosis: a cardiovascular magnetic resonance study. Journal of Cardiovascular Magnetic Resonance 2015 17(Suppl 1):Q71.

\section{Submit your next manuscript to BioMed Central} and take full advantage of:

- Convenient online submission

- Thorough peer review

- No space constraints or color figure charges

- Immediate publication on acceptance

- Inclusion in PubMed, CAS, Scopus and Google Scholar

- Research which is freely available for redistribution

Submit your manuscript at www.biomedcentral.com/submit
C Biomed Central 